\title{
CORRIGENDUM
}

\section{Cryoprotective effects of low-density lipoproteins, trehalose and soybean lecithin on murine spermatogonial stem cells - CORRIGENDUM}

Peng Wang, Ying Li, Xiao-chen Hu, Xiao-Li Cai, Li-Peng Hou, Yan-Feng Wang, Jian-Hong Hu, Qing-Wang Li, Li-Juan Suo, Zhi-Guo Fan and Bo Zhang

doi:10.1017/S0967199412000378 Published by Cambridge University Press, 14 September 2012.

The Authors apologise for the error in the name of 'Northwest A \& F University', which appears underneath the author names, and also in the footnote, both on page 1 of the article.

It should read as follows:

College of Animal Science and Technology, Northwest A\&F University, Yangling, Shaanxi 712100, P.R. China.

\section{Reference}

Peng Wang, Ying Li, Xiao-chen $\mathrm{Hu}$, Xiao-Li Cai, Li-Peng Hou, Yan-Feng Wang, Jian-Hong Hu, Qing-Wang Li, Li-Juan Suo, Zhi-Guo Fan and Bo Zhang. Cryoprotective effects of low-density lipoproteins, trehalose and soybean lecithin on murine spermatogonial stem cells. Zygote. Published by Cambridge University Press, 14 September 2012. doi:10.1017/S0967199412000378 